\title{
The Solution to the Mind-body Problem: The Complete Reduction of the Human Mind to Matter, Namely to the Brain
}

\author{
Michael Prost \\ Philosophy Bottrop, 46244 Bottrop, Germany
}

Copyright $(2017$ by authors, all rights reserved. Authors agree that this article remains permanently open access under the terms of the Creative Commons Attribution License 4.0 International License

\begin{abstract}
The mind-body problem asks about the specifics of the human mind and its interaction with the body. We will show that what makes the human mind unique is the advanced human language. We will illustrate that some mutations in Homo sapiens enabled us to develop a sophisticated language, which was the basis for the development of advanced abstract thinking. We will show how language and thinking are realized in the brain by neural connections. That leads to the conclusion that the human mind is our language-representing neural network, and can indeed be completely reduced to matter. Materialism and reductionism are true. Actually, the solution of the mind-body problem also explains human consciousness. Human consciousness is the ability of humans to include verbal thinking in conscious processing. A comparison shows that prominent theories of consciousness such as Global Workspace Theory (GWS), Higher Order Thought Theories (HOT) and Integrated Information Theory (IIT) are a subset of our explanation. But all other theories are also applicable to animals with brain, miss the specifics of human consciousness and do not deliver an explanation on a neural level.
\end{abstract}

Keywords Mind, Consciousness, Language, Verbal Thinking, Neural Correlates, Hard Problem

\section{What Is the Status of the Mind-body Problem?}

One of the most puzzling questions of mankind is the question about the special character of the human mind and human consciousness. This is the mind-body problem of philosophy. So far neither philosophers nor natural scientists have been able to find a satisfying solution for this problem. The common assumption for all attempts is naturalism, which includes substance monism. Naturalism is closely connected with physicalism, materialism and reductionism which try to reduce the human mind and human consciousness to matter. Matter again is part of physical explanations. In principle it should be expected that naturalism, physicalism, materialism and reductionism are basically the same. Nevertheless there exists the perplexing position that most philosophers consider themselves naturalists but reject physicalism, materialism and reductionism. Most philosophers insist that on the one hand the human mind is indeed based on a natural thing, namely the brain, but that on the other hand it is not possible to reduce the mind to pure matter. They insist that the mind is something very special and unique which cannot be described by natural sciences alone. Let's have a look at some current positions.

One of the most prominent proponents of physicalism is Jaegwon $\operatorname{Kim}[1,2,3]$. Kim's starting point is a separation of the mind-body problem into two different problems, mental causation and consciousness. With the term consciousness he denotes phenomenal experiences or qualia. Kim makes his distinction between mental causation and consciousness / phenomenal experiences for a good reason; his answer is that mental causation can indeed be reduced to physics whereas phenomenal experiences cannot. His argumentation for his first claim lies in the assumed closeness of the physical world. In the view of naturalism and the simultaneous refusal of substance dualism only physical causes can cause physical effects. Consequently, Kim argues, mental states that represent mental causation must be reducible to physical states. In other words, there must exist special physical configurations of the brain that represent a special kind of mental state and that cause actions of the body. Because these configurations are physical states, the respective set of mental states must be explainable by physics. Phenomenal experiences such as 'feeling pain' or 'seeing red' do not cause any physical effects. Consequently, Kim assumes that consciousness / phenomenal experiences are not reducible to physics. Another proponent of physicalism is David 
Papineau who goes one step further $[4,5]$. Papineau agrees with Kim that mental causation is reducible to physics. Papineau also admits that the fact that consciousness / phenomenal experiences do not play a causal role is the reason why it is more difficult to associate them with physical states. On the other hand Papineau doesn't see why this should be impossible. It might be difficult in reality to ever find the neural correlates for phenomenal experiences, but it is theoretically possible. Actually, the issue is that Papineau merely argues on a conceptual level without giving a concrete explanation of how mental states can be reduced to physical states.

The latter is the reason that not everybody agrees. A lot of philosophers are not convinced and still see an 'explanatory gap', a term that was first introduced by Joseph Levine [6]. Two famous examples for the alleged explanatory gap were given by Thomas Nagel in his paper 'What is it Like to Be a Bat' [7] and by Frank Jackson in his paper 'What Mary Didn't Know' [8]. Today the 'explanatory gap' is very ambitiously defended by David Chalmers $[9,10,11,12]$ who called the problem the 'hard problem'. The hard problem concerns phenomenal experiences, which this group also refers to as consciousness. Chalmers considers problems such as explaining mental causation or cognition 'easy', while explaining in physical terms what it is like 'to feel something' is characterized as 'hard'. The general strategy in Chalmers' argumentation is the conceivability of zombies. Zombies are considered to be creatures that are physically identical to humans but lack consciousness. Consequently so argues Chalmers - consciousness cannot be explained physically, that means materialistically. Again the mind-body problem is not solved.

These three examples demonstrate some principle issues. First, the terms used in the discussion are not unambiguous. Therefore we will first provide some definitions everybody can agree upon. Especially the term 'consciousness' needs some clarification. In addition it is necessary and helpful to ask if the term 'consciousness' can even be applied to animals. The same goes for the terms 'thinking', 'feeling' and 'mind'. Secondly, it seems futile to discuss the mind-body problem mainly on a theoretical, conceptual level. If the assumption of philosophy is naturalism this includes substance monism. If the assumption is substance monism then there is no location in space-time where a non-material mind or non-material phenomenal experiences can exist. The question cannot be 'Is it possible to reduce mind and consciousness to matter?' Rather the question can only be 'How can mind and consciousness be reduced to matter or how does the brain form mind and consciousness?' Here we will demonstrate the final solution to the mind-body problem based on naturalism. This solution will also explain human consciousness.

\section{Clarification of Terms}

As mentioned above, all considerations here are based on naturalism. Consequently, we have to explain what naturalism means. Naturalism is the view that all phenomena existing in the universe can be explained in a natural way. This should be understood that everything can be explained by natural sciences. The fundament of natural science is physics. Physics claims that the universe began with the big bang and that in the big bang only one kind of substance was created, namely matter. ${ }^{1}$ In the further development of the universe matter formed all phenomena existing in the universe, such as stars, planets, animals, plants and finally humans. Consequently, every aspect of humans is based on matter and can be explained by physics. This leads to the fact that every aspect of humans can vice versa be reduced to matter. Physics claims that the world is causally closed and that there cannot exist any gaps. If the definition of naturalism is indeed that the universe can be explained by natural sciences then naturalism, physicalism, materialism and reductionism are identical. Then it is evident that the human mind can be reduced to matter and the only open question is how this can be done. Now it has to be emphasized that there are philosophers who consider themselves naturalists but reject reductionism. They dispute that physics can explain everything, especially humans and the human mind. Consequently they deny the causal closure of nature. This position might be respectable, but it is not justified to call it naturalism. The position assumes that something is missing in the current knowledge of natural science. Even if this were really the case it cannot be known if this missing part is really natural. Therefore this view should not be called naturalism. Furthermore, we have to claim that all positions which call themselves naturalism but are not in agreement with our definition are in reality not naturalistic. We will come back to Chalmers' zombie argument at the end of the forth chapter.

One of the major reasons why the mind-body problem has so far remained unsolved is the confusion concerning the different terms used in the debate. Some of the critical terms are 'consciousness', 'mind', 'mental state', 'mental causation', 'phenomenal experiences', 'thinking', 'feeling' and 'qualia'. First, we have to provide a proper definition of the term 'consciousness', which is obviously the basis for the mind. It has to be emphasized that consciousness is nothing unique to humans. On the contrary, consciousness is a state of the brain that all animals with brains possess. ${ }^{2}$ What's more, consciousness is one of the most important features without which evolution would never have been able to develop the large variety of animal species existing today. It is very well known that most of the actions a brain performs are unconscious. For example, the brain regulates all basic

1 Today, physics assumes that there exist three different kinds of matter, namely ordinary matter, dark matter and dark energy. (Because Einstein has shown the equivalence of matter and energy, dark energy is also considered to be matter). But dark matter and dark energy do not have any effects on earth. Consequently, physics claims that everything on earth can be explained by ordinary matter.

2 At least for all animals with a further developed brain, which include all vertebrates. 
life functions such as heartbeat, breathing, digestion or moving unconsciously. Also reflexes are executed unconsciously. The characteristics of reflexes are that the brain initiates an action of the body based on an internal (e.g. pain) or an external (e.g. danger) sensation. This action is performed immediately and without any further consideration. But evolution 'found out' that it would be beneficial if the brain were able to initiate actions based on a bigger set of sensations and that it would be beneficial if the brain were able to initiate actions based on a longer and more complex set of brain processes.

To illustrate this principle we will use the example of nutrition of an amoeba and of a leopard. When an amoeba accidentally touches any kind of food in its environment the food is consumed through its cell wall. That is a complete act of nutrition based on reflexes. This is entirely different and much more complex for a leopard. A leopard feels the internal sensation of hunger. The leopard starts to prowl looking for impala. After the leopard spots some impala he checks the environment. He 'knows' that for his approach he needs some cover, and that he has to pay attention to the wind direction. In addition, he has to estimate the distance to his prey. He has to take into account his own acceleration ability and the acceleration ability of the impala. All the described actions are controlled by the consciousness of the leopard's brain. First the brain reacts to some internal sensation (hunger). Consequently the brain activates a search process. Now the brain processes all incoming visual sensations and compares these sensations with information about possible prey. This information has been stored in the brain of the leopard, when the leopard's mother taught her child what and how to hunt. After identification of a possible prey the brain of the leopard activates some different programs that have also been stored in the leopard's brain during the education process. Now the brain collects additional information about surroundings and covers, about wind direction and about distance to the prey. When the hunting program in the brain comes to the conclusion that all parameters fit together the brain initiates the attack. This example illustrates how consciousness as a state of the brain works and gives its bearer totally different new opportunities compared to a simple creature like an amoeba. This explanation shows that consciousness is not unique to humans; it applies at least to animals with a brain as well. Here our definition of consciousness is that consciousness is a capability of the brain to initiate actions based on a broad set of sensations after a lengthy complex process. ${ }^{3}$ Actually, this definition is approved by neuroscience and should be acceptable for philosophy as well.

Next let us define the term 'mental state'. In a broader sense every state of the brain could be considered to be a mental state. But it makes sense to restrict the term 'mental states' only to conscious states of the brain. Very often, philosophers use the term 'mental state' as a distinguishing

3 Again, this definition might be constrained to vertebrates feature between animals and humans. But again, even the assignment of mental states solely to humans is not completely correct because animals have mental states, too. Per definition a mental state is a state of the brain, which might cause some action of the body or which might be a phenomenal experience. A mental state that causes some body action leads to mental causation. Consequently mental causation is a process in which a special configuration of the brain causes some actions of the body. Now we should emphasize again that we are not talking about humans only, we are still talking about animals with a brain as well. So obviously animals also have the ability of mental causation.

Animals also have phenomenal experiences. Phenomenal experiences are considered to be described by questions such as 'What is it like to feel pain?' or 'What is it like to see red?' We use these examples for phenomenal experiences on purpose. These examples show that there are two different kinds of phenomenal experiences. The first kind is caused by internal sensations, which may be pain, hunger and any other emotion or feeling. These phenomenal experiences very often cause actions. Again we use our hunting leopard. The entire hunting process is initiated by an internal phenomenal experience, namely by hunger. Phenomenal experiences caused by internal sensations can cause actions, but they don't necessarily have to do so. If we feel a piercing pain in our hand, because we have touched a sharp object, we immediately withdraw the hand. If we have a moving pain in one of our joints we can't do much. Maybe we rest. Maybe we just feel it. The other kinds of phenomenal experiences are caused by external sensations, e.g. by a red object or noise. Actually, this usually doesn't cause a direct action; it is more part of an information gathering process. This information might be used later in different mental processes to lead to mental causation. As mentioned above, the term 'qualia' is often used in philosophy to denote phenomenal experiences. The purpose of the use of the term 'qualia' is to demonstrate that phenomenal experiences have a special quality for every individual who experiences a phenomenon. A good example again is pain. There are very many different kinds of pain and we can assume that every individual experiences pain in a different way. What we see now is that phenomenal experiences are identical with sensations. The term, 'phenomenal experiences', was invented to illustrate that some phenomena - either internal or external - create experiences which are individualistic and qualitative. But this is indeed the characteristic of all sensations; this is nothing special. The individuality and qualifiability of sensations as the basis of conscious processing is the reason for different actions. Hunger must initiate a different reaction than pain. Seeing red must initiate a different reaction than seeing green. The question how phenomenal experiences can be explained in a natural materialistic way now transforms in the question how sensations can be explained naturalistically and materialistically. We have also mentioned that sometimes philosophers use the term 'consciousness' for phenomenal experiences and deny the 
reducibility. Now we see that this argument is equivalent to the deniability of the reducibility of sensations. Obviously, this doesn't make sense in naturalism. Again, we have to emphasize that everything said here applies to animals as well which means that all kinds of arguments which use the non-reducibility of phenomenal experiences against materialism and reductionism such as those of Nagel, Jackson, Levine and Chalmers would have to be applied to animals as well. But we have shown here that phenomenal experiences are just internal and external sensations. As we will show later, neuroscience can easily reduce all sensations to matter, namely to neural connections in the brain. Consequently all those kinds of arguments are wrong.

We now still have to give definitions for the terms 'thinking' and 'feeling'. When we analyze the leopard's hunting process we have to concede that animals also have feelings and also can think. It is probably indisputable that indeed animals can have feelings. When we look at feelings such as hunger or pain we have called these situations phenomenal experiences. Consequently feelings are a subset of phenomenal experiences, namely the subset that represents internal sensations. What's about thinking? The activities a leopard has to perform during a hunt must indeed be considered a kind of thinking. These activities are logical and rational. The leopard combines different external sensations in a complex and lengthy process to eventually initiate an action. Because this kind of thinking is based on sensations we call this kind of thinking 'sensory thinking'. Animals indeed have the capability for sensory thinking. So we have to concede that animals also can think. ${ }^{4}$ Summarizing we can say that animals with a brain also have consciousness and mental states leading to mental causation. Animals with a brain have phenomenal experiences or qualia, and they can think.

This leaves us with the term 'mind'. Really unique for humans is the possession of a highly advanced declarative language. We know from the barking of dogs or the singing of birds that animals also have a kind of language that they use for communication. But that kind of language is only rudimentary and is called signal-language. As the name suggests, signal-language is used to share information by producing some simple signals. Obviously, human language can do a lot more. ${ }^{5}$ Eventually we can clarify what is unique to the human mind and to human consciousness is: it is the ability to speak and to enhance thinking and feeling to a completely different level through language. Because this new kind of thinking by humans involves words we call it 'verbal thinking'. The use of language and verbal thinking now enhance all the capabilities we have previously identified in animals. Human consciousness is now not only

4 It has to be emphasized that this is an (arbitrary) definition. The definition is based on the fact that the behavior of animals is in many cases rational and logical which are both considered to be characteristics of thinking. If thinking were necessarily connected to language - what we will explain in the next paragraph - then animals would consequently not be able to think. 5 The decisive feature between signal language and declarative language is the second level of abstraction. We will explain this a little bit later. the ability of the brain to process internal and external sensations, human consciousness now can also integrate verbal constructions. Whereas the leopard will immediately start his hunting process when he feels hunger, humans can decide that they would like to eat later. They can reflect on their internal sensations, their phenomenal experiences. That also applies to the other kind of phenomenal experiences, those caused by external sensations. Humans can report about them, describe them and reflect on them. Whereas the leopard has to follow its original program to get food, humans can decide that they want to change their diet. For humans mental causation must not necessarily be based on sensations, it can also be based on reflections. Through language humans develop convictions, desires, beliefs, intentions and can act on reasons. Whereas the leopard has to eat impala all his life, humans can decide that they want to be vegetarians for ethical reasons. Humans can reflect about their feelings and are free to influence and maybe change them through reflection. It is obvious that this list can be extended almost infinitely. Human mental states now also contain advanced declarative language. Consequently, the human mind is the capability of the brain for verbal thinking. Some might object here that human thinking is more than just using language. But this objection lacks any justification. Of course, we have outlined that there is also sensory thinking. And because humans are also animals we are capable of sensory thinking and indeed we use sensory thinking in a lot of situations, even if considering an intellectual problem, for example a mathematician trying to solve a geometrical problem. The mathematician might stare at some geometrical configurations and all of a sudden recognize a correlation. He has found the solution to his problem. And yet, the solution is not accessible until he brings it into a linguistic expression. Not until the solution is linguistically described is the solution complete. That is also the case if someone has a problem and makes decision about the problem emotionally without much reflection. As neuroscience has found out even in this case subconscious reflections using language occur leading to a decision. The reflection process was just not completely conscious. But in any case the solution can now be described verbally; the solution is available in language. Summarizing we can say that reflections which are considered to be the characteristic capability of the human mind need always language to reach the extraordinary level only found in humans. Especially abstract thinking is only possible by using language. Actually, we experience the connection between language and thinking every moment we are awake. While thinking we speak silently to ourselves. Without language we cannot think. This justifies describing the human mind as the capability of the brain for verbal thinking, per definition using language. The connection between thinking and language will still become still more transparent a little bit later when we describe the development of language.

In summary we can say that a lot of features normally attributed only to humans in philosophical discussion also 
exist in animals. At least animals with brain have consciousness, have phenomenal experiences and qualia and can initiate mental causation. Animals with a brain have feelings and are capable of sensory thinking. We must strongly assume that all phenomenal experiences and qualia in animals are comparably individualistic as they are in humans. We could also ask 'What is it like for an animal to feel pain?' or 'What is it like for an animal to see red?' That directly shows that the assumption of an explanatory gap would have to be extended to animals as well. If this should be avoided the question arises whether the additional quality that is added to phenomenal experiences by language might give reason to assume an explanatory gap only for humans. We will show that this is not necessary and not possible. Again, we have to emphasize that consciousness is not special to humans, and neither are phenomenal experiences. Both exist in all animals with brain and are identical among humans and other animals on a biochemical cellular level. ${ }^{6}$ The only difference between humans and other animals with brains is the declarative human language. That is the reason why it makes sense to link the terms 'human consciousness' and 'mind' to declarative language.

There are two tasks. First, we will show how humans developed language and advanced thinking and that this can be explained in a very natural way. Secondly, we will show how indeed every mental entity is realized physically in the brain. This explanation then eventually will show how phenomenal experiences are realized by physical states. And this explanation will also show how the human mind and human consciousness can be described in physical terms; how the human mind can be reduced to matter, namely to the brain.

\section{The Development of Human Language and Human Thinking}

Naturalism assumes that a process called evolution produced a group of animals called primates and amongst the primates there evolved a family called great apes that besides orangutans, gorillas and chimpanzees includes a subspecies called Homo. The true patriarch of modern humans was Homo erectus who evolved from her predecessors around two million years ago in Africa. Already Homo erectus left Africa and spread to Asia and Europe. In Asia and Europe Homo erectus developed amongst other species into Homo neanderthalensis. Homo neanderthalensis was the last non-human ${ }^{7}$ Homo species that lived together on earth with humans and went extinct 30,000 years ago. Some 200,000 to 150,000 years ago, Homo erectus in Africa evolved to Homo

6 The task of demonstrating how consciousness is created on a biochemical cellular level is part of neuroscience and cannot be achieved by philosophy. 7 When we use the term 'non-human' here, we want to emphasize that only Homo sapiens is considered to be 'really' human, because only Homo sapiens developed the ability of human thinking. We will prove this a little bit later. sapiens. Homo sapiens still exist as the modern human. We will now show how human language developed in a natural way and that indeed human language is the decisive feature for the explanation of the human mind and human consciousness.

The intriguing questions are how humans developed the human language and at what point we can consider the human kind of thinking different from that of animals. So far the question of how humans developed the human language has been addressed from the wrong perspective. Here the author brings in the decisive new clue. So far the question was always how evolution changed the human brain in order to develop human language. Without any doubt there has to be a necessary level of brain development in order to develop language and thinking. But the author is convinced that this necessary level is already achieved in chimpanzees. That claim can be illustrated by the case of the female chimpanzee Washoe, born 1965, deceased 2007, who was the first non-human being that was able to communicate with humans in a typically human way. This was possible because the American anthropologist Roger Fouts [13] taught Washoe the American Sign Language (ASL) that is used by deaf-mute people. Indeed, Washoe learned some hundred different signs and was able to communicate with Fouts. What's more, Washoe was even able to combine different signs into meaningful sentences. And what's still more, Washoe had an adopted chimpanzee son, Loulis, to whom Washoe taught ASL - with both chimpanzees ultimately able to communicate using ASL! This example proves at least that even chimpanzees have the cognitive abilities for advanced language and advanced communication. ${ }^{8}$

The example of Washoe now leads us directly to the paradigm change that is necessary to explain the development of human language and of human thinking. The decisive prerequisite for the development of human language is not modifications of the brain, but instead some evolutionary anatomic changes in the human 'speaking apparatus'. The term, 'speaking apparatus', is supposed to include all body parts of the head that play a role in the formulation of spoken words. Here Homo sapiens has experienced major changes compared to chimpanzees, but also compared to older kinds of Homo. Two of these major changes are the lowering of the larynx and the enlargement of the pharynx [14]. These two evolutionary changes gave Homo sapiens a previously unknown ability to create different sounds. The author claims that the decisive factor for the development of a sophisticated language is not only a special ability of the brain, but also the ability to create a large variety of sounds. We can illustrate the principle if we

8 Now the objection may come up that humans are much more advanced in their ability to use ASL and therefore must have a different cognitive ability than chimpanzees. That is absolutely true. But it has to be taken into consideration that the human brain evolved with the evolution of the human language over the last 150,000 years. Therefore modern humans now have superior cognitive abilities compared to chimpanzees. The question is whether chimpanzees would have had a similar development if they had developed the ability to speak. 
assume that we could hypothetically only create the five vowels. Of course, five vowels can create an infinite number of combinations. That means one would be able to create an infinite number of different terms, which is sufficient to create any sophisticated language. But the caveat is that the human brain or any other brain would not be able to remember all these combinations and thus would realistically reduce the possibility of sophisticated language, even if it were theoretically possible. It would certainly be feasible for the brain to associate different meanings with the combinations 'AUI', 'OUI', 'EIA' or even 'AIEU'. But if the combinations 'AUIEEAUOEI' or 'OIUAEEOIU' are formed, the brain will not be able to remember the possible meaning of those combinations. The development of any language would be limited to the degree we see in animals' signal-language. But if the range to create different sounds is extended to the variety we see in humans by being able to produce syllables with vowels and consonants, then obviously the human brain is able to remember these different combinations with their assignment to different meanings.

We know that Homo sapiens developed from the African line of Homo erectus about 200,000 to 150,000 years ago and we can be pretty sure that the origin of the development of human language lies in today's Botswana. There and at that time the aforementioned mutations of the speaking-apparatus occurred and Homo sapiens began to develop an advanced declarative language. We know this because every language has some elements called 'phonemes', which are small units of any language that give terms different meanings. For example, the terms 'kiss' and 'kill' have different meanings because the phoneme 'ss' is replaced by the phoneme 'll'. Every language has a certain numbers of phonemes. Studies [15] have found out that the number of phonemes decreased with the propagation of Homo sapiens. The language with the most phonemes is !Xóõ, a language spoken in Botswana. The language with the least phonemes is Hawaiian, spoken on Hawaii. Actually, Hawaii was the last piece of earth where humans settled. If one now follows the path of human propagation one sees that the number of phonemes decreases the farther away Homo sapiens settled from her origin in Africa, namely in Botswana. It took Homo sapiens about 140,000 to 190,000 years until human language and the ability to think was so far developed that we had the intellectual capacity to start agriculture and livestock farming and to settle down ${ }^{9}$. With settlements, agriculture and livestock farming Homo sapiens was able to assign different tasks to different members of the group. Before that, all men went hunting and all women were in charge of preparing food and raising the children. With the dedication of different tasks to different members of the group a new level in the development of human language and of human knowledge began. From that point on, it took humankind about 12,000 years to bring people to the moon. In

9 That happened about 10000 B.C. conclusion we can say that a sufficiently developed brain is necessary for the development of a sophisticated language. But that is not the decisive factor because we can assume that Homo erectus most likely had such a brain and even chimpanzees seem to have it. The decisive factor is that the anatomy of the speaking apparatus is able to create a large variety of different sounds. After this critical mutation happened to Homo sapiens she could start to develop an advanced language and advanced thinking. ${ }^{10}$

Now we have to investigate the development of the human language in some more detail. The basis of every language, even of signal languages, is abstraction. Animals have sounds to alarm the other members of a group about a danger. If we imagine a group of impala, the danger could be a leopard or a cheetah. So both situations, either threatened by a leopard or threatened by a cheetah, are obviously different because leopards and cheetahs are different. But both situations have something in common, namely the threat of being caught by a predator. So even though both situations are different, the common scenario makes it reasonable to develop just one signal for both situations, because both situations require the same reaction, namely sudden escape. The development of a signal or a sound for a set of similar situations can be considered to be the first level of abstraction. We must assume that Homo erectus did indeed also have sounds to denote situations and objects. But as we pointed out before the language of Homo erectus remained rudimentary because Homo erectus was not able to produce enough different sounds with her speaking apparatus to reach the next level of a developed language and the level of advanced thinking.

This level started with the second level of abstraction. An example for the second level of abstraction is the term 'fruit'. The first level of abstraction is to denote different fruits with different sounds, e.g. 'mango', 'banana' and 'pineapple'. Maybe Homo erectus was already aware that mango, banana and pineapple had something in common. ${ }^{11}$ All objects were edible, all objects were sweet and all objects contained a relatively large amount of fluid. But Homo erectus was not able to develop a new term, namely 'fruit', for these types of objects, because she just wasn't able to produce enough different sounds. But Homo sapiens was, due to the mutations of the speaking apparatus! That new second level of abstraction now paved the way for the development of abstract terms, like 'fruit'. At the first level of abstraction, every term is connected with an imagination. We can imagine an object and we can imagine a situation like the threat from a predator. For the second level of abstraction, we don't have a direct imagination any more. If we hear the term 'fruit', we know general characteristics of fruit and we can have the imagination of some examples for fruit but we

10 It should be mentioned that in the course of the development of language some modifications of the brain were also needed, namely a higher neural transmission speed. We will come to this point later.

11 That is just an example. Actually, obviously Homo erectus didn't eat many fruits. 
do not have any direct imagination of the term 'fruit' anymore. After the second level of abstraction began to be developed, we can assume that one of the further developments was probably the development of numbers, and following that the development of simple mathematics. In this way the second level of abstraction also paved the way for the development of time as part of language. With enough sounds and enough terms it was now possible to make a distinction between past, present and future. With the development of time it was also possible to discover and to describe causality. Our example of the hunting leopard has shown that animals, and definitely Homo erectus, are / were able to act based on causality. But we have shown that these actions are based on imaginations / sensations, therefore we called it sensory thinking. The second level of abstraction, with the development of numbers and the development of time as part of language, now gave Homo sapiens the ability to figure out and to formulate causal connections based on language. And of course, now Homo sapiens was able to pass every causal discovery on to other members of her group and to her descendants. We can now summarize that the basis for every advanced kind of thinking is the development of an advanced language with the ability of a second level of abstraction. We now can define that the particularity of human thinking, of the human mind and of human consciousness is the capability for advanced language and for abstract thinking. As already mentioned above, we can call this new kind of thinking 'verbal thinking'. Even if someone were to dispute the claim of the author that mutation of the speaking-apparatus are the reason for the development of language and thinking there remain some undeniable facts. First, an adequate speaking-apparatus is necessary for the development of an advanced language. Secondly the development of the second level of abstraction is necessary for advanced thinking. The paradigm change is that not the human mind developed language, but that language developed the human mind.

\section{Reducing the Human Mind and Human Consciousness to the Brain}

Let us examine some basic explanations of how the brain works. These principles are very well known and described in great detail by many authors, for example by Gerhard Roth [16]. The brain contains two kinds of cells, neurons and glia cells. The glia cells insulate the neurons from each other and play a role in the nourishment of the neurons. What we call brain activity is performed by the neurons. ${ }^{12}$ Generally speaking, neurons form connections between each other and send signals along these connections. We speak of neural connections and neural processes. Eventually signals are sent

12 Actually, there exist several different kinds of neurons, but the difference does not play a role here because we are not considering the cell level of neural connections. to muscles that trigger muscle actions. As we have already mentioned above, most of the neural processes occur unconsciously. Because we want to reduce the human mind and human consciousness to matter, we will concentrate on conscious brain activities. First let us describe what happens when we see something. Actually, this would be one kind of phenomenal experience. As we know this process also occurs in animals. We see some kind of image with our eyes. That means that there are signals created in the retina that send signals to the brain, namely to a region called the cortex where all conscious processes take place. ${ }^{13}$ In the cortex the signals from the eyes form a kind of neural connection that we call a neural pattern. Such a neural pattern in the cortex represents an object or a configuration of objects of the external world. To some degree these neural patterns are conserved in the brain. The next time we see something, again a neural pattern is formed. Now the brain performs a neural function that compares the new pattern with old neural patterns representing images seen before. If the cortex finds an old neural pattern that is very similar to the new neural pattern the cortex now 'knows' that the new image is known and has already been seen before. During the growing up of a human or any other animal the cortex 'learns' to distinguish different neural patterns and to assign to these different patterns different objects or configurations of objects. Actually, the same principle applies to all kind of sensations or phenomenal experiences.

The next step in development, again in humans as well as in a lot of animals, is to connect some kind of reaction to the visual neural patterns. For example, a baby impala has to learn that a leopard is a predator, and if that predator comes too close the impala has to make an escape. To the neural pattern of a leopard there will be formed a neural connection to a different pattern that means 'dangerous when too close', and to that pattern there will be formed a neural connection to a neural pattern that initiates the escape. This is the general functionality of consciousness. Animals with brains create neural patterns in the cortex that are initiated by sensations, either external sensations like images or internal sensations like hunger. The phenomenal experiences 'feeling hunger' or 'seeing red' are nothing but a special configuration of neural patterns. Naturally imbedded in the cortex is a neural functionality that compares newly formed neural patterns with old neural patterns and builds a 'memory'. In the process of growing up, the animal learns to act based on the current sensations. That means new neural connections are formed in the cortex along which signals are transferred. These new neural connections represent new neural processes. Based on the current sensations these neural processes can trigger some actions. This is mental causation. Now we see that mental causation is a neural process that causes the body to move. Above we have described the

13 More precisely: All signals of external sensations first go to a part of the midbrain, the thalamus, and then are transferred to different specified areas of the cortex, such as the visual cortex or the auditory cortex. 
sensory thinking of a hunting leopard. On the basis of certain sensations (hunger, searching for prey, identification of prey, estimating distance to prey) the neural hunting process is initiated and the leopard goes after the impala. In conclusion, all actions of the leopard are based on neural processes in the brain, namely in the cortex. We have learned that the same scheme applies to humans. ${ }^{14}$ And we have also learned that animals have features such as phenomenal experiences and mental causation, which were originally thought to be exclusive to humans. We have seen that these features are neural patterns and neural processes. That means we have shown how phenomenal experiences or qualia and mental causation can be explained by the brain and be reduced to matter.

Is there something different in humans that might not be reducible? We have connected the human mind to human language. Consequently we will illustrate what happens when we learn the language as a child. Actually, the same principles apply later to every kind of conversation. First we hear some sounds with our ears. Our ears send a signal to the brain. The signals from the ears form some special neural connections that we already know as neural patterns; here these patterns are 'audible neural patterns'. We can call these audible neural patterns 'language-patterns'. That means here an audible neural pattern or a language-pattern represents a word or a combination of words. Additionally we learn to connect the neural language-patterns with imaginations. That is the first major difference to all other creatures. When we see an object, e.g. a banana, we compare the neural pattern created by the image with old neural patterns and recognize a banana. That is the same procedure as with animals. But in addition, we immediately connect the visual pattern with a second pattern representing the term we learned to denote that object. When humans see a banana, there is a second neural connection formed beside the visual pattern, namely to the language-pattern. As we are aware, that process also works vice versa. When we hear the word 'banana' a neural connection is immediately formed that connects the language-pattern for banana with the visual pattern for banana. Automatically we know what a banana is and what a banana looks like. Again, a certain level of abstraction is involved, because all bananas are different but they are all similar. The kind of thinking involved adds language-patterns to objects and classifies objects by language, which is why we call this kind of thinking verbal thinking. In the second step of learning the language an additional type of neural language pattern is now created, namely that which initiates our speaking apparatus to create meaningful sounds, the words. This type can be denoted as 'speak-patterns', a second kind of neural language-pattern. Consequently in humans every primary neural pattern that was created by external or internal sensations is simultaneously connected to two additional neural

14 We have used the example of visual sensations. But the same scheme applies to all other senses as well. language-patterns. ${ }^{15}$

We have emphasized above that the second level of abstraction does not connect words directly with imaginations anymore. For example when we hear the word 'fruit' we don't have a direct connection to an image such as when we hear the word 'banana'. Indeed we have a kind of blurred imagination and we might have a variety of imaginations of examples for fruits. But if we hear a different abstract word, such as the number 'one', there is no image connected at all. But with the neural pattern for 'one' there arise a lot of different neural connections that immediately let us know what 'one' means. With human language rules developed as to how these abstract entities, words, could be connected. Most of the rules are based on the definition of the words. When we learn a language we also learn all the rules for how to combine words and these rules are realized as neural patterns in our cortex. When we speak our cortex combines patterns of words that we know in a way that is permissible and guided by the different sets of neural patterns that represent the rules of meaningful language. The resulting language-patterns then initiate our speaking apparatus to speak. The decisive feature of the human brain is the ability to develop neural patterns that represent words and that represent the rules that allow the combination of language-patterns. We will illustrate this capacity with one of the most intriguing features of human thinking, namely mathematics. When Homo sapiens developed language, she invented numbers. ${ }^{16}$ Numbers are stored as neural patterns in the cortex, and after some experience with counting, Homo sapiens figured out that she arrived at identical numbers whether she counted up or added the numbers. Therefore she stored some new neural patterns, now representing the sum of numbers. Instead of, for example, counting first to four and after that five more times resulting in nine, she now knew immediately the sum of four plus five, also resulting in nine. Later she invented number systems and rules within these number systems that allowed her to add numbers basically up to infinity. Again we have to be aware that all these mathematical entities and all the mathematical rules are stored as neural patterns. The cortex is able to apply these rules as neural processes to neural patterns, mathematical entities, in order to produce new neural patterns, now representing mathematical results. We can emphasize here that whenever we practice special human thinking we use our language. When we think we speak

15 As mentioned before, the simultaneous neural connection of three different neural patterns now required a modification of the cortex, namely a higher neural transmission speed. This was achieved by a higher myelination.

16 Actually, it has to be emphasized that animals cannot count. Indeed, this can be fatal for leopards. A leopard can distinguish between one person and several persons, but he just can't count. If hunters hunt a leopard they usually hang some bait in a tree. If a leopard takes the bait, the hunters build a blind not far from the bait. Before nightfall the hunters approach the blind. Because the leopard is probably already watching the bait he also sees the hunters. He sees a group approaching and a group leaving. What he does not realize is the fact that one hunter stayed in the blind. If the leopard now approaches the bait assuming that no hunter is around anymore the one hunter can kill the leopard. 
silently to ourselves. Maybe at the beginning of the development of the language only loudly spoken words were possible. But during the evolution of language Homo sapiens learned that she can 'speak silently' to herself. So even if thinking is always connected to language it is not necessary to speak out loud during thinking. But even more: We can never 'not think' as long as we are awake, as we always experience our brain continuously producing some kind of thought. We speak permanently to ourselves! Actually, what we do is connect neural speak-patterns in neural processes. In other words: verbal thinking can create thoughts!

So far we have found out that there are two kinds of thinking: sensory thinking and verbal thinking. We have shown that animals have the capability for sensory thinking, but only humans have advanced language and the capability for verbal thinking based on abstraction. We have shown that both kinds of thinking are realized in the brain by neural processes that connect neural patterns. The uniqueness of verbal thinking is that in addition to neural patterns that are created by sensations - the primary neural patterns - a second set of neural patterns is created that represents terms for the primary patterns. Verbal thinking is the ability to process these language-representing neural patterns in neural processes and to form new language-representing neural patterns. The second level of abstraction goes even further and now creates neural language-patterns not based on imagination anymore, but based on other neural language-patterns. These findings now enable us to describe how the human mind can be reduced to matter.

But before we do so, we will include the emotional effects in our explanation. A different meaning of the term, 'consciousness', now comes into play. In our definition consciousness was a capability of the brain to act based on a broad set of sensations after lengthy complex processes. Accordingly, human consciousness includes language and abstract terms in these processes. But sometimes the term 'human consciousness' is also used to add the emotional aspect to the term 'human mind'. Can we reduce this kind of human consciousness to matter, to the brain, as well? Again we start by looking at animals. As we pointed out, animals also have feelings and emotions. As we have seen, these feelings, which represent internal phenomenal experiences, create neural patterns. What now is specific to humans? That is the fact that emotional neural patterns do not necessarily create immediate actions, but that humans can also process these emotions through verbal thinking. Humans can associate terms with all kinds of feelings. They can also associate qualitative characteristics to these feelings. Not only do they know that they have some kind of pain and can say so, but they also can describe the type of pain and its intensity. Again we have to remember that these phenomenal experiences are qualia and now we see that qualia in humans add neural language-patterns to neural phenomenal patterns. Humans can also use their verbal thinking, again based on language and abstraction, to find reasons for feelings and emotions. If humans have found causes for special feelings or emotions that they don't want to have they can think about measures to eliminate the causes and consequently eliminate the unwanted feelings and emotions. If we now put together the verbal thinking about causality and the verbal thinking about emotions, it leads us to the term 'self-reflective I'. The 'self-reflective I' is the part of humans that reflects about the person herself, about her background, about her characteristics, about her talents and capabilities, about her emotions, about her relations with other people and about her future, her intentions and her desires. As we see now this all happens by using language which means that everything is realized in the brain by neural processes and neural patterns. Finally we can solve the mind-body problem: The human mind is our language-representing neural network. Human consciousness is the capability to include verbal thinking in conscious processing. The actions initiated in conscious processing through verbal thinking are thoughts realized as language-representing neural patterns. Everything that is specific to the human mind is realized by neural connections that are associated with language. Everything that is specific to human consciousness is also realized by neural connections that are associated with language.

Finally we have shown that materialism, reductionism and physicalism are correct. Now we can come back to Chamber's use of zombies. The assumption of naturalism is that the big bang only created matter. Matter later evolved into all the known structures of the universe. Thus a priori every structure including mental states must be reducible to matter. In addition, the laws of physics require that systems with identical physical characteristics show the same behavior. If zombies are now assumed to have the same physical features as humans they must also have the same mental states. If this is denied and mental states are considered not be reducible to matter mental states must exist in a different universe. That immediately raises the question where this other universe is, how it was created and how it interacts with our universe. Every account that denies the reducibility of mental states to matter assumes a priori substance dualism and the existence of a different universe. As we have mentioned above this is not naturalism anymore. This example shows that sometimes philosophers consider themselves naturalists but in reality they are not. As we have already mentioned above it can be stated in general that all objections of philosophers against materialism and reductionism are always based on a misunderstanding of naturalism and physics. Indeed we have to concede that the question 'What is it like to feel something?' suggests more than there is. We have shown that phenomenal experiences are identical with sensations. It is common neuroscientific knowledge that sensations are realized in the cortex as neural patterns. This leads to the conclusion that phenomenal experiences are just certain neural configurations. The hard problem is a pseudo problem. The difficulty to describe phenomenal experiences verbally does not prevent us from reducing them to matter, namely to brain states. 


\section{How Does the Presented Solution Fit Together with Current Philosophical Theories of Consciousness?}

We now want to look at some current philosophical theories about consciousness and compare them with our solution. Maybe the most popular theories are 'Global Workspace Theory', 'Higher-Order Thought Theories' and 'Integrated Information Theory'. The 'Global Workspace Theory' (GWT) was introduced and later further elaborated by Bernard Baars [17, 18, 19]. GWT assumes that consciousness is a process, in which a lot of participants (of the brain) are involved. GWT recognizes that there is always only one conscious process at a time. Simultaneously there are many subconscious processes happening which concur for consciousness as well. GWT also recognizes that many different regions of the brain are involved in one conscious process. GWT also recognizes that the purpose of consciousness is to initiate voluntary actions. And finally, GWT recognizes that conscious processing always takes place serially. But Baars makes a concession to the proponents of the hard problem and admits that GWT might not be complete in order to close the explanatory gap. As we see easily, the general concept of GWT is in great agreement with our explanation because both rely on the discoveries of neuroscience. GWT describes the discoveries of neuroscience in philosophical language. Neuroscience has found out that there is only one serial conscious process, neuroscience has found out that different parts of the brain are involved in one conscious process, and neuroscience has found out that subconscious processes are also involved in conscious processing and sometimes concur for consciousness. GWT merely describes these discoveries in a more tentative language. But what GWT does not achieve is to deliver a reduction of consciousness or the human mind to neural correlates. And what GWT also misses completely in the description of human consciousness is the critical role of human language. The description of consciousness given by GWT can also be applied to animals. As we have shown here, that is correct but misses the characteristics of human consciousness. That is also the reason why Baars concedes that the hard problem might not be solvable by GWT. In conclusion, we can say that our explanation is in great agreement with GWT but that our explanation goes several steps further than GWT and eventually delivers a complete explanation of human conscious even on a neural level.

'Higher-Order Thought Theories' exist in different versions. Proponents include David Rosenthal, Josh Weinberg and Hakwan Lau [20, 21], Paula Droege [22] and Edmund Rolls [23]. The general idea is that there are first-order mental states, which are consciously processed in a higher order. First-order mental states could be all kinds of sensations that became conscious when they are assessed by higher-order thoughts. 'Higher-order Thought Theories' (HOT) are also based on the discoveries of neuroscience. It is known that various different areas in the cortex are involved in conscious processing. There are some areas where sensations create initial neural connections. We have called these first-order neural connections neural patterns. It is also known that there exist in the cortex some additional associative areas that are involved in conscious processing of sensations. We have called these appropriate neural connections neural processes. In this sense, HOT at least describes some aspects of consciousness in a correct way and is in complete agreement with our explanation. One could also argue that HOT is a further elaboration or an addendum to GWT. But again, HOT only describes some discoveries of neuroscience with philosophical language. HOT is also applicable to animals. HOT also misses completely the importance of human language for human consciousness, and HOT does not give any explanations on a neural level.

'Integrated Information Theory' is a concept introduced and later been further developed by the neuroscientist Gulio Tononi $[24,25,26]$. 'Integrated Information Theory' (IIT) starts with the fact that consciousness including phenomenal experiences surely exists and asks what the conditions were for physical realization. IIT realizes that conscious experiences are structured (every conscious experience has distinct elements), that conscious experiences contain information, that the information is integrated into one element of conscious experience and, of course, that every conscious experience is distinct from each other conscious experience. An important deduction for any physical correlate of consciousness is the requirement of cause-effect structure. IIT is indeed in good agreement with many insights of neuroscience. Obviously, consciousness is closely related to the ability of the brain to process integrated information. On the other hand, a loss of consciousness (sleep, coma) goes together with less information processing. One characteristic of IIT is indeed that IIT assumes the possible reduction of mental states to brain states, i.e. IIT assumes that materialism and reductionism are correct. Consequently IIT is in full agreement with our explanation. But again, also IIT is applicable to animals. IIT has not taken into account the importance of human language for human consciousness, and IIT does not give any explanation about neural correlates. Yet it can be emphasized that if human language is integrated into IIT the predictions of IIT might become more relevant still. That is absolutely evident because the additional layer of language in human conscious processing evidently generates much more information. In this sense it can be formulated that indeed human consciousness differs significantly from animals' consciousness because human consciousness is connected with much more integrated information.

Summarizing we can say that some of the current philosophical theories of consciousness, e.g. GWS, HOT and IIT, are in good agreement with the explanation of human consciousness given in this publication. Moreover, GWS, HOT and IIT are partial descriptions of our explanation on a higher, more abstract level of language, so to speak on a meta-level. But actually, the abstract level makes them very disputable because the explanation on a neural level is missing. The advantage of our explanation is that the human 
mind and human consciousness are explicitly reduced to neural correlates.

\section{Conclusions}

We have shown here that the human mind and human consciousness can indeed be completely reduced to matter, namely to the brain. The decisive paradigm change is the discovery that human language is the salient difference to other animals, especially to other great apes. Language became possible in Homo sapiens because of anatomic mutations of the speaking apparatus such as the lowering of the larynx and the enlargement of the pharynx. We know that the previous species of Homo did not have the necessary anatomy to develop an advanced language. We must also assume that they were consequently restricted in their intellectual capacities. With language Homo sapiens developed the second level of abstraction, which must be considered the critical point to distinguish animals' thinking, which we called sensory thinking, from human thinking, which we called verbal thinking.

Subsequently we have shown that everything - including feelings and phenomenal experiences, sensory thinking and verbal thinking - is realized in the brain through neural connections. Finally we have been able to solve the mind-body problem and explain human consciousness. The human mind is our language-representing neural network and human consciousness is the capability of humans to include verbal thinking in conscious processing. Human mind and human consciousness are completely reduced to matter, namely to the brain. Physicalism, materialism and reductionism are true within naturalism.

But indeed, there remains a real 'hard problem'; neuroscience has to find out what the neural connections really look like and how different areas of the brain work together to eventually initiate actions.

\section{REFERENCES}

[1] J. Kim. Mind in a Physical World. Thorverton: A Bradford Book, MIT Press, 2000

[2] J. Kim. Physicalism or Something near Enough. Princeton: Princeton University Press, 2005

[3] J. Kim. Philosophy of Mind. Boulder: Westview Press, 2010

[4] D. Papineau. Introducing Consciousness. London: ICON Books, 2000

[5] D. Papineau. Thinking about Consciousness. New York: Oxford University Press, 2002

[6] J. Levine. Materialism and Qualia: the Explanatory Gap. Pacific Philosophical Quarterly 64, 354-361, University of
Southern California, 1983

[7] T. Nagel. What Is it Like to Be a Bat?, Philosophical Review LXXXIII, 4, 435-50, 1974

[8] F. Jackson. What Mary didn't Know, Journal of Philosophy, 83, 5, 291-295, 1986

[9] D. Chalmers. Explaining Consciousness: The Hard Problem, Journal of Consciousness Studies 2 (3), 200-219, 1995

[10] D. Chalmers. The Conscious Mind: in Search of a Fundamental Theory. New York: Cambridge, MA: Cambridge University Press, 1997

[11] D. Chalmers. 'The hard problem' and 'Moving forward on the problem of consciousness'. In J. Shear (ed.), Explaining

[12] D. Chalmers. Phenomenal Concepts and the Explanatory Gap. In: T. Alter and S. Walter (eds.), Phenomenal Concepts and Phenomenal Knowledge: New Essays on Consciousness and Physicalism'. New York: Oxford University Press, 2006

[13] R. Fouts. Next of Kin: What Chimpanzees have Taught Me about Who We Are. New York: William Morrow and Company, 1997

[14] A. Ghazanfar, D. Rendell. Evolution of Human Vocal Production, Current Biology, Volume 18 (11), R457-R460, 2008

[15] Q. Atkinson. Phonemic diversity supports a serial founder effect model of language expansion from Africa. Science, Vol. 332, No. 6027, 346 - 349 Oxford University Press, 2012

[16] G. Roth. The long evolution of brains and minds, New York: Springer. 2013.

[17] B.J. Baars. A Cognitive Theory of Consciousness. Baars, B. J.(1997), In the Theater of Consciousness. New York, NY: Oxford University Press, 1988

[18] B.J. Baars. The conscious access hypothesis: Origins and recent evidence. Trends in Cognitive Sciences, 6 (1), 47-52, 2002

[19] B.J. Baars. The conscious access hypothesis: Origins and recent evidence. Trends in Cognitive Sciences, 6 (1), 47-52, 2002

[20] D. Rosenthal, J. Weisberg. Higher-order theories of consciousness. Scholarpedia 3 (5): 4401, 2008

[21] H. Lau, D. Rosenthal. Empirical support for higher-order theories of conscious awareness. Trends in Cognitive Sciences 15 (8): 365-373, 2011

[22] P. Droege. Higher-Order Theories of Consciousness. Internet Encyclopedia of Philosophy, 2014

[23] E. Rolls. Emotion Explained. New York: Oxford University Press, 2005

[24] G. Tononi. An information integration theory of consciousness. In: BMC Neurosci. 5, 42ff, 2004

[25] G. Tononi. Consciousness as integrated information: a provisional manifesto. Biol Bull. 215(3), 216-242, 2008

[26] G. Tononi. Phi: A Voyage from the Brain to the Soul. New York: Pantheon Books, 2012 